\title{
Near-infrared fundus autofluorescence imaging in a case of photic maculopathy
}

\author{
Alberto Neri ${ }^{1}$, Alessandra Protti ${ }^{2}$, Monica Camparini ${ }^{2}$, Claudio Macaluso ${ }^{2}$ \\ University of Parma, Parma, Italy \\ Correspondence: Alberto Neri. Address: University of Parma, Parma, Italy. Email: neri.mail2@gmail.com \\ Received: February 6, 2014 \\ Accepted: February 19, $2014 \quad$ Online Published: February 28, 2014 \\ DOI : $10.5430 /$ ijdi.v1n1p28 \\ URL: http://dx.doi.org/10.5430/ijdi.v1n1p28
}

\section{Abstract}

Herein we describe the characteristics of scanning laser ophthalmoscope imaging (SLO), and particularly of near-infrared fundus autofluorescence modality (IRAF), in photic maculopathy. IRAF visualizes selectively the melanin granules contained in the cells of the Retinal Pigment Epithelium (RPE), which are normally localized in the apical portion of RPE cells, a favorite target of the photic damage to the retina. In the present work we report a three-month follow-up of a case of photic maculopathy, showing that the retinal alterations found by IRAF precisely matched the retinal anomalies found by macular optical coherence tomography, and had a parallel evolution. We think that IRAF could represent a useful imaging modality, together with more conventional imaging, in the management of photic maculopathy.

\section{Keywords}

Photic maculopathy, Near-infrared fundus autofluorescence, Scanning laser ophthalmoscopy.

\section{Case presentation}

Photic maculopathy (PM) ${ }^{[1]}$ was diagnosed in a 28 year-old female patient complaining of bilateral central scotoma and decline in visual acuity lasting seven days, with onset during a video-recorded dance show. High power arc flood lamps were used for illumination of the dance floor. Medical history reported only chronic migraine in treatment with amitriptyline $40 \mathrm{mg} /$ day. The patient expressed informed consent to the publication of the present report.

Best-corrected distance visual acuity (BCVA) measurement with ETDRS panels (Early-Treatment Diabetic Retinopathy Study), macular optical coherence tomography (OCT) (Cirrus HD-OCT ${ }^{\circledR}$, Carl Zeiss Inc., Jena, Germany), and retinal imaging with scanning laser ophthalmoscope (SLO) (HRA2, Heidelberg Engineering, Heidelberg, Germany) were performed at the 7th, 30th, and 90th day after the onset of symptoms. Retinal pictures were obtained by SLO using three different imaging modalities: infrared (IR, $820 \mathrm{~nm}$ ), blue-autofluorescence (BAF, obtained with excitation at 488nm and a filter at $500 \mathrm{~nm}$ ), and near-infrared-autofluorescence imaging (IRAF, obtained with excitation at $790 \mathrm{~nm}$ and a filter at $810 \mathrm{~nm})^{[2]}$.

Figure 1 shows the clinical and instrumental findings at the baseline and during the follow-up of the patient. At presentation BCVA was 20/32 in both eyes, with a central positive scotoma wider in the right eye. Fundoscopy at presentation showed pale yellow-grey small spots at the foveal centers. With the SLO the foveal anomalies appeared as dark spots with IR imaging, and as hypo-autofluorescent spots with IRAF and BAF imaging (less evident with blue- than 
with IR- autofluorescence). In the right eye a small hyper-autofluorescent spot was also present at the temporal edge of the hypo-autofluorescent spot in IRAF. OCT of the macula showed outer retinal anomalies localized at the foveola, consisting of disruption of the Verhoeff membrane (junction between the photoreceptor outer segment and RPE apical processes) and underlying hyporeflectivity of inner aspect of the retinal pigment epithelium layer (RPE).

Oral supplementation of antioxidants, macular pigments and $\Omega 3$-fatty-acids (Azyr Mega, SIFI, Catania, Italy), was prescribed. The follow-up showed a gradual improvement of visual acuity with BCVA of 20/16 and disappearance of the central scotoma at the 90th day. The OCT showed progressive reformation of a normal Verhoeff membrane, consistently with the improvement in visual acuity, and significant regression of the hypo-reflectivity in RPE layer. Corresponding gradual regression of the foveal anomalies was observed with the SLO; IRAF imaging was particularly useful to monitor the retinal changes at the fovea (see Figure 1).
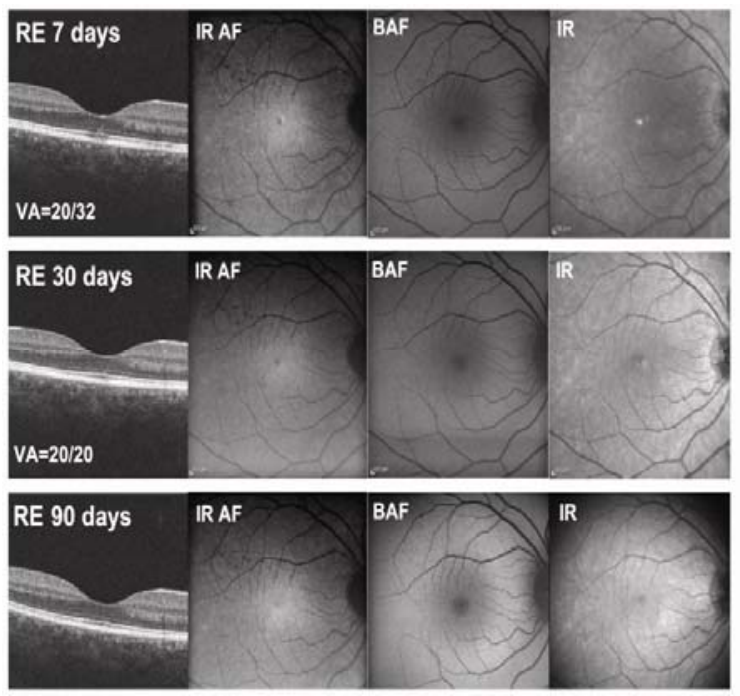
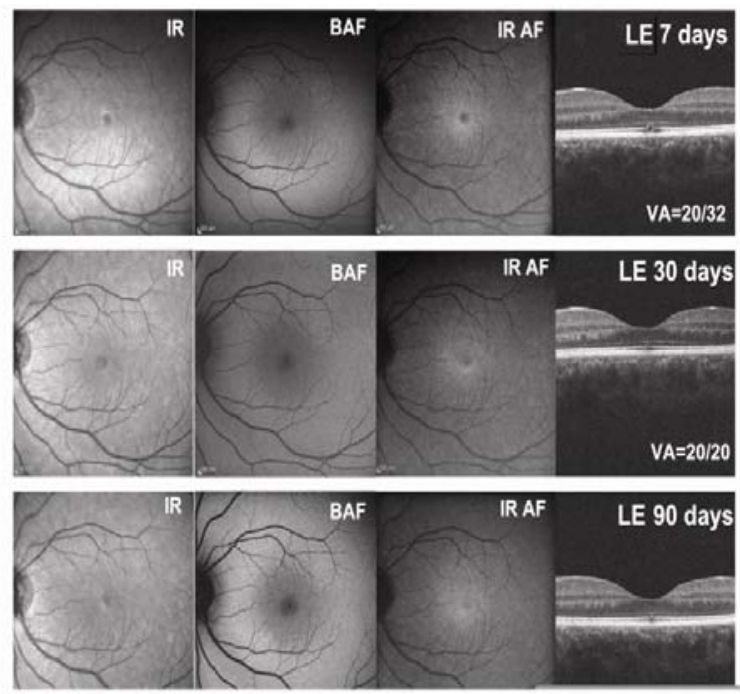

Figure 1. optical coherence tomography (OCT) and scanning laser ophthalmoscope (SLO) retinal imaging of the patient at presentation ( 7 days from the onset of the symptoms) and during the follow-up (30 and 90 days from the onset) are shown. Best-corrected distance visual acuity (VA) at each step of the follow up is shown in the figure. The images of the right eye (RE) are shown on the left, the left eye (LE) is shown on the right. Pictures of normal right and left eyes are shown at bottom for comparison. SLO imaging of the retina was obtained with three modalities: infrared (IR), blue-autofluorescence (BAF) and infrared-autofluorescence (IR AF).

OCT of the macula showed outer retinal anomalies localized at the foveola, consisting of disruption of the Verhoeff membrane and underlying hyporeflectivity of the inner aspect of the retinal pigment epithelium layer (RPE). With SLO, the foveal anomalies appeared as dark spots with IR imaging, and as hypo-autofluorescent spots with IRAF and BAF imaging. The hypo-autofluorescence was more evident with IR- than with blue- autofluorescence. In the right eye a small hyper-autofluorescent spot was also present at the temporal edge of the hypo-autofluorescent spot in IRAF at presentation. Both OCT and SLO anomalies showed a progressive regression to normality during the follow-up.

\section{Discussion}

Photic maculopathy (PM) occurs when an intense light irradiation overwhelms the tolerance of the retinal tissue, damaging the macula ${ }^{[1]}$. The diagnosis of PM is based on the history and the observation of characteristic macular anomalies by ophthalmoscopy and optical coherence tomography (OCT) ${ }^{[1,3,4]}$.OCT has high sensitivity in the diagnosis and follow-up of photic maculopathy, showing in high resolution some characteristic alterations of RPE-photoreceptors 
complex ${ }^{[3,4]}$.Interestingly, we observed that the retinal pictures obtained by SLO, in particular with IRAF modality, reflect precisely the outer retinal alterations highlighted by the OCT.

Blue and near-infrared fundus autofluorescence visualize different structures of the retina ${ }^{[2]}$. BAF derives mainly from lipofuscin, which is localized in the basal portion of the RPE cells, while IRAF visualizes selectively the melanin granules in RPE cells, which are normally localized in the apical portion of RPE cells ${ }^{[2]}$.Also, the pattern of blue and infrared autofluorescence of the normal macula is different: the center of the fovea, in fact, is normally hypo-autofluorescent in BAF, while IRAF has the maximal intensity at the center of the fovea and decrease eccentrically ${ }^{[2]}$. The physiological central BAF hypo-autofluorescence does not correspond to a reduced amount of lipofuscin in the center of the macula, but depends on the higher absorption of blue light (excitation light) by the yellow macular pigment ${ }^{[2]}$. BAF showed a reduced ability to detect the RPE changes visualized on OCT scans in the present case. This may be due to the fact that RPE changes induced by the photic injury did not influence significantly the amount of lipofuscin in the RPE. Moreover, BAF seems inherently less useful in bringing detailed information about the RPE in the foveolar zone, as it tends to be masked by macular pigment absorption. Differently, IRAF images of the retina in our patient clearly showed a central hypo-autofluorescent spot, exactly matching with the zone of OCT anomalies. Hence, it is conceivable that the hypo-autofluorescent zone in IRAF may reflect a damage to the apical portion of RPE cells, where melanin is accumulated, and that the corresponding OCT alteration is the hypo-reflecting RPE band.

\section{References}

[1] Mainster MA, Turner PL: Photic Retinal Injuries: Mechanisms, Hazards, and Prevention. In: Retina. Volume 2, 5th edn. Edited by Ryan SJ, Schachat AP, Wilkinson CP, Hinton DR, Sadda S, Wiedemann P. Philadelphia: Saunders/Elsevier. 2013; $1555-1563$.

[2] Kellner U, Kellner S, Weinitz S: Fundus autofluorescence (488 NM) and near-infrared autofluorescence (787 NM) visualize different retinal pigment epithelium alterations in patients with age-related macular degeneration. Retina. 2010; 30(1): 6-15. http://www.ncbi.nlm.nih.gov/entrez/query.fcgi?cmd=Retrieve\&db=PubMed\&dopt=Citation\&list_uids=20066766.

[3] Comander J, Gardiner M, Loewenstein J: High-resolution optical coherence tomography findings in solar maculopathy and the differential diagnosis of outer retinal holes. Am J Ophthalmol. 152(3): 413-419 e416. http://www.ncbi.nlm.nih.gov/entrez/query.fcgi?cmd=Retrieve\&db=PubMed\&dopt=Citation\&list_uids=21708377.

[4] Huang SJ, Gross NE, Costa DL, Yannuzzi LA: Optical coherence tomography findings in photic maculopathy. Retina. 2003; 23(6): 863-866. http://www.ncbi.nlm.nih.gov/entrez/query.fcgi?cmd=Retrieve\&db=PubMed\&dopt=Citation\&list_uids=14707841. 